\title{
RELACIÓN ENTRE ENGAGEMENT Y LAS CREENCIAS DE AUTOEFICACIA EN LOS TRABAJADORES
}

\section{RELATIONSHIP BETWEEN ENGAGEMENT AND SELF-EFFICACY BELIEFS IN WORKERS}

María Fajardo Sarmiento, Marianela Iroz Bortolotto, Debla López Talavera, Seima Mirón Hernández. Universidad de Granada.

\section{RESUMEN}

Hoy en día, si se quiere tener una alta productividad laboral, es necesario crear un alto nivel de compromiso organizacional (work Engagement) de los empleados con la empresa. Es necesario estudiar las variables psicológicas que favorecen el "work Engagement". Según la Teoría Social Cognitiva de Bandura, la creencia de eficacia predice a largo plazo la satisfacción familiar y las conductas sanas (Bandura, 2004). En la presente investigación analizamos la autoeficacia y su relación con el Engagement organizacional, para descubrir si realmente esta variable puede ser mediadora en el Engagement. Para ello se ha medido, en 105 trabajadores de diferentes sectores, el nivel de autoeficacia percibida mediante General Self Efficacy Scale y el nivel de work engagement utilizando el Utrecht Work engagement Scale. Esperábamos obtener a mayores niveles de autoeficacia mayor nivel de engagement. Al mismo tiempo, medimos el engagement de estos trabajadores teniendo en cuenta variables como sexo, ámbito laboral y nivel de estudios.

PALABRAS CLAVE: work engagement, creencias de autoeficacia, bienestar laboral, estudio correlacional.

\begin{abstract}
Nowadays, if you want to have a high labor productivity, it is necessary to create a high level of organizational commitment (workEngagement) of employees with the company. You need to study the psychological variables which favor the "workEngagement". According to Bandura's Social Cognitive Theory, the efficacy belief predicts family satisfaction and health behaviors (Bandura, 2004). In this study we analyze the self-efficacy and its relationship to organizational Engagement, to discover if really this variable may be a mediator in the Engagement. To do this, we was measured in 105 workers from different areas, the level of self-efficacy by General Self Efficacy Scale and level of workengagement using Workengagement Utrecht Scale. We expected to have higher levels of self-efficacy to higher levels of engagement. At the same time, measure the engagement of these workers taking into account variables such as gender, workplace and educational level.
\end{abstract}

KEY WORDS: work engagement, self efficacy belief, work-related wellbeing, correlational study.

\section{INTRODUCCIÓN}

Para rendir al máximo en un mundo cada vez más competitivo, en todos los aspectos vitales en general, y en el ámbito laboral en particular, los trabajadores deben tener asegurado no sólo la preparación académica y laboral, sino también aspectos 
psicológicos individuales y sociales que los lleven a experimentar satisfacción y sentido de logro. Las empresas tienen una gran responsabilidad en este sentido, y deben esforzarse para poder potenciar al máximo las capacidades de sus trabajadores.

Actualmente nos vemos inmersos en un mundo cambiante, a nivel social, laboral y tecnológico. Vivimos a un ritmo acelerado, y para hacer frente de manera óptima a estas exigencias es preciso que los trabajadores nos sintamos capaces de nosotros mismos, que creamos que nuestras competencias personales y profesionales son adecuadas para hacer frente a las diversas circunstancias del día a día. Por ejemplo, autores como Cherniss $(1980,1993)$ señalan que en el desarrollo del burnout (desgaste en el trabajo), la falta de confianza en las propias competencias es un factor crítico.

Tradicionalmente, la psicología había venido ocupándose de aspectos negativos y patológicos de la conducta humana. Más recientemente, Seligman propuso estudiar al ser humano desde una perspectiva positiva, enfocándose en su funcionamiento óptimo (Seligman, Steen, Park y Peterson, 2005). De esta manera, la Psicología Positiva centra su objetivo en potenciar las capacidades del ser humano.

Llevando esta postura al mundo de las organizaciones, la psicología se interesa en aspectos como la creatividad, la iniciativa, la responsabilidad y el compromiso del trabajador con su empresa. La Psicología Positiva Organizacional es definida como "el estudio y aplicación de recursos y competencias humanas, que pueden ser medidas, desarrolladas y gestionadas con el objetivo de mejorar el desempeño en las organizaciones" (Salanova, Martínez y Llorens, 2006). En definitiva, se trataría de conseguir, en última instancia, el bienestar laboral y la máxima satisfacción y rendimiento del trabajador.

Para definir el bienestar laboral, se han desarrollado muchas teorías y se han estudiado diversas variables. Dos de ellas son el compromiso laboral (Work Engagement) y el estado de desgaste (Burnout) (Bakker y Oerlemans, 2011). Ambos constructos psicológicos reflejan el grado de conexión psicológica que la persona tiene con su trabajo.

El primer autor en definir el Work Engagement fue Khan en 1990, que lo describió como qué tan "enganchado" está el empleado con el trabajo que realiza. Otra definición hace referencia al grado en que los empleados se encuentran involucrados, comprometidos, entusiasmados y apasionados sobre su trabajo (Macey y Schneider, 2008). Es un indicador de motivación intrínseca por el trabajo, un estado cognitivo afectivo persistente en el tiempo. Se daría cuando los niveles de energía en el trabajo son altos, se observa una gran persistencia y hay un deseo de esforzarse.

Este constructo estaría formado por tres componentes. En primer lugar el componente físico (vigor), definido por altos niveles de energía física y predisposición para invertir esfuerzos. En segundo lugar el componente emocional (dedicación), expresado en altos niveles de entusiasmo, orgullo e inspiración en lo que se hace. Por último el componente cognitivo (absorción), que incluye la concentración y la sensación de que el tiempo pasa rápido (Schaufeli, Salanova, González-Romá y Bakker, 2002).

Como cabe esperar, el grado de implicación teniendo en cuenta estos tres componentes, se puede ver afectado por diversas variables, tanto internas como externas al trabajador. Según Schaufeli y Salanova (2011), cuando el empleado percibe un desequilibrio entre lo que él aporta a la empresa con su trabajo y lo que obtiene a cambio, el vigor puede convertirse en agotamiento, la dedicación en cinismo 
y la absorción en ineficacia profesional. Identificar estos déficits a tiempo permitiría realizar intervenciones para que no se vean afectadas la productividad de la empresa y el bienestar de sus trabajadores.

Luthans, Youssef y Avolio (2007) hablan del capital psicológico como un recurso con que cuenta la persona y que predice el bienestar laboral. Dentro del capital psicológico se encuentran: la creencia de autoeficacia, el optimismo, la esperanza y la resiliencia (capacidad de permanecer fuerte en momentos difíciles). En este estudio nos centraremos exclusivamente en la autoeficacia como predictora del grado de implicación laboral.

El autor más influyente en el estudio de la autoeficacia ha sido Albert Bandura. Este autor define dicho concepto como "los juicios de cada individuo sobre sus capacidades, con base a los cuales organizará y ejecutará sus actos, para alcanzar el rendimiento deseado" (Bandura, 2002). En realidad este concepto no hace referencia a los recursos con que cuenta objetivamente la persona, sino a la opinión que tenga sobre ellos, su percepción subjetiva acerca de si son suficientes y útiles para hacer frente a los problemas y alcanzar sus objetivos.

Si las creencias de autoeficacia son altas, es más probable que la persona esté motivada y comprometida, desempeñándose eficazmente en sus actividades, y afectando por lo tanto a su bienestar laboral de manera positiva.

Según Martínez y Salanova (2006), las personas que se consideran poco eficaces en el afrontamiento de las demandas del entorno exageran sus deficiencias y las dificultades potenciales del medio. Esta percepción negativa de sí mismos genera estrés y distrae la atención, y dificulta la utilización de los recursos disponibles (ya que no los perciben). Por el contrario, las personas que se perciben eficaces centran sus esfuerzos en las demandas que la situación les pueda plantear.

Por otro lado, Bandura sostiene que la autoeficacia también determina la manera en cómo consideramos a los obstáculos. Las personas con alta autoeficacia los ven como impedimentos u obstáculos superables, aumentando su nivel de esfuerzo y perseverancia. Las personas con baja autoeficacia, por otra parte, se convencen a sí mismas que es inútil esforzarse ya que no lograrán su objetivo, y acaban abandonando.

Teniendo en cuenta todo lo mencionado anteriormente, en este estudio se plantea analizar la relación existente entre las creencias de autoeficacia y la implicación laboral de un grupo de trabajadores. Para esto hemos averiguado en primer lugar cuál es el nivel de las creencias de autoeficacia de los participantes. En segundo lugar, necesitamos conocer su nivel de implicación en el trabajo (Work engagement). Por último, analizar si realmente existe una relación entre ambos constructos. Es decir, si las creencias de autoeficacia pueden influir en el nivel de engagement en el trabajo.

Si esto es así, el conocimiento de dicha relación tendría importantes aplicaciones prácticas para las empresas, ya que podrían aplicar programas para incrementar las creencias de autoeficacia de sus trabajadores, y así conseguir maximizar su desempeño, logrando una mayor implicación y satisfacción laboral.

\section{Hipótesis:}

$\mathrm{H} 1$ : Las creencias de eficacia se asocian positivamente con el engagement, es decir, existirá una relación positiva entre las variables engagement y autoeficacia de manera que a mayor autoeficacia generalizada (en el trabajo) mayor engagement laboral. 
H2: La media de las puntuaciones de las mujeres en todas las dimensiones del engagement será equiparable a la media de puntuaciones la de los hombres.

H3: Los participantes pertenecientes al ámbito laboral privado tendrán medias más altas en todas las dimensiones del engagement que los del ámbito público.

H4: Los participantes con estudios universitarios tendrán unas puntuaciones medias en las dimensiones del engagement mayores a las de los participantes sin estudios universitarios.

\section{METODOLOGÍA}

\section{Participantes:}

Para esta investigación se contó con la participación voluntaria y anónima de 105 trabajadores de la provincia de Granada, seleccionados al azar de diferentes empresas y sectores, entre otros: hostelería, educación y funcionarios públicos. La media de edad de los participantes es de 39,0667 años (DT=11,40198), siendo de 20 años la edad mínima y 65 años la edad máxima de los participantes.

La muestra estaba compuesta por 42 hombres (40\%) y 63 mujeres $(60 \%)$.

La media del tiempo trabajado de la muestra es de 12,2499 años (DT=9,59318), siendo el mínimo tiempo trabajado 0,20 años y el máximo 38 años.

Se dividió el ámbito laboral de los participantes en dos grupos: público (administraciones públicas, funcionariado) y privado.

Se contempló tres valores de la variable nivel de estudios siendo estos: básicos (hasta EGB o educación elemental), medios (BUP/COU/FP/ESO/Bachiller) y universitarios (Diplomaturas, Licenciaturas e Ingenierías).

\section{Instrumentos:}

Se construyó un cuestionario que incluía las siguientes medidas:

- Datos sociodemográficos: edad, sexo, sector de la empresa, nombre de la empresa, tiempo trabajado en la empresa, puesto que desempeña en la empresa, nivel de estudios.

- Escala de Work Engagement de Utrecht (Utrecht Work Engagement Scale, UWES), (Shaufeli et al., 2002), en la adaptación española de Salanova et al. (2000). Esta escala está compuesta por 17 ítems y comprende tres dimensiones: Vigor, Dedicación y Absorción. Para el vigor se dispone de 6 ítems (un ejemplo de ítem sería "soy fuerte y vigoroso en mi trabajo"), para la dedicación 5 (un ejemplo sería "estoy orgulloso del trabajo que hago"), y para absorción 6 (un ejemplo sería "estoy inmerso en mi trabajo"). Estos ítems se responden por medio de siete opciones de respuesta en una escala Likert de 0 a 6 . Se han obtenido los siguientes coeficientes alpha de Cronbach para las dimesiones: Vigor, $\alpha=.803$; Absorción, $\alpha=.80$; Dedicación $\alpha=.85$.

- Escala de Autoeficacia General (General Self Efficacy Scale, GSE), de Jerusalem y Schwarzer (1992). Esta prueba evalúa el sentido general de la creencia de autoeficacia, principalmente en cuanto a su capacidad de enfrentar problemas diarios. 
Contiene 10 ítems para los que los participantes deben responder su grado de acuerdo con cada enunciado en una escala tipo Likert con 4 alternativas de respuesta (1= "Falso" a 4= "Cierto"), logrando una puntuación mínima de 10 y un máxima de 40 puntos. Sus propiedades psicométricas han sido ampliamente establecidas tanto en población no española como española. Algunos ejemplos de ítems son: "Tengo confianza en que podría manejar eficazmente acontecimientos inesperados"; "Puedo encontrar la manera de obtener lo que quiero aunque alguien se me oponga". Se ha obtenido un alpha de Cronbach de .816 .

\section{Procedimiento:}

Se pidió la colaboración voluntaria y anónima a los participantes. Una vez que éstos accedieron, se les administró el cuadernillo que contiene los cuestionarios. Sólo se respondió a aquellas preguntas formuladas acerca de los datos sociodemográficos, no a las formuladas acerca de las escalas. Los participantes rellenaron el cuestionario y posteriormente fueron recogidos dándoles las gracias por su colaboración.

\section{Análisis de datos:}

Los análisis realizados fueron en primer lugar análisis descriptivos de las diferentes variables a analizar, como medias, desviaciones típicas y consistencias internas (alpha de Cronbach). Posteriormente se calculó los índices de correlación de Pearson entre las distintas dimensiones del engagement y la autoeficacia generalizada. Por último, para corroborar las hipótesis $\mathrm{H} 2, \mathrm{H} 3$ y H4, se realizó las diferencias de medias tStudent. Para realizar estos análisis se utilizó el paquete estadístico IBM SPSS Statistics versión 21 de prueba de 14 días.

\section{RESULTADOS}

Los porcentajes en cuanto al ámbito laboral de los participantes, público $(\mathrm{N}=50$, $47,6 \%)$ o privado $(\mathrm{N}=55,52,4 \%)$, quedan reflejados en el siguiente gráfico:

Gráfico 1: Porcentaje de participantes según el ámbito laboral.

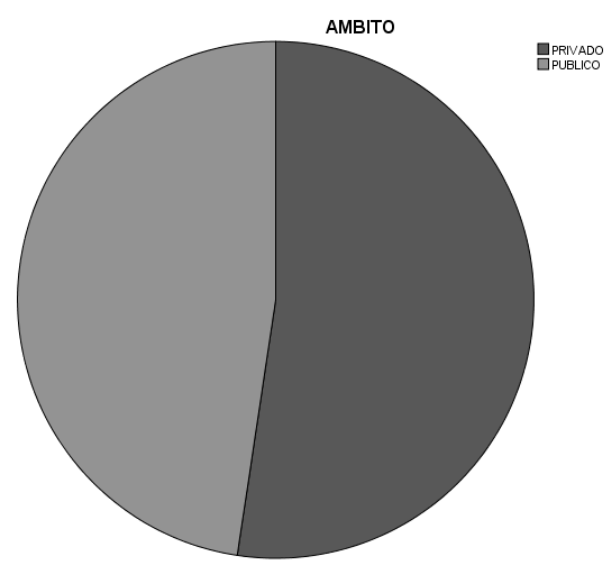

La distribución de participantes atendiendo a la variable nivel de estudios siendo es la siguiente: básicos $(\mathrm{N}=6,5,7 \%)$, medios $(\mathrm{N}=35,33,3 \%)$ y universitarios $(\mathrm{N}=64,61 \%)$ 


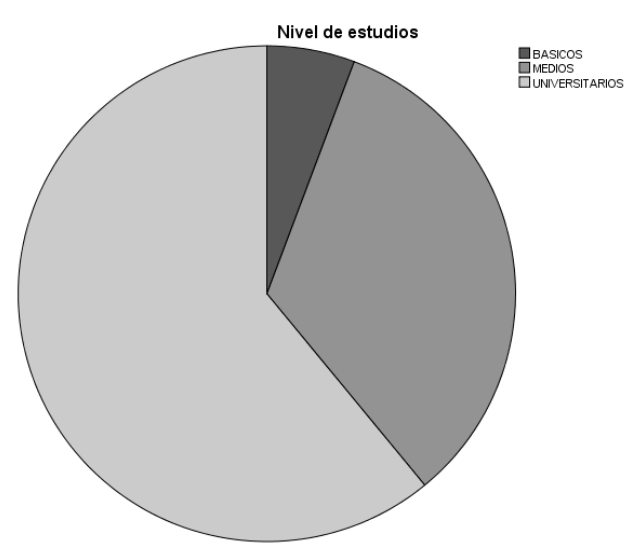

Gráfica 2: Porcentaje de participantes según nivel de estudios.

Los estadísticos descriptivos de los datos sociodemográficos tales como edad y tiempo trabajado (indicados ambos en unidades de tiempo anuales) son recogidos en la siguiente tabla:

Tabla 1. Estadísticos descriptivos de las variables de edad y tiempo trabajado.

\begin{tabular}{|c|c|c|c|c|c|c|}
\hline & \multirow{2}{*}{$\begin{array}{c}\mathrm{N} \\
\text { Estadístico }\end{array}$} & \multirow{2}{*}{$\begin{array}{l}\text { Mínimo } \\
\text { Estadístic } \\
\text { o }\end{array}$} & \multirow{2}{*}{$\begin{array}{l}\text { Máximo } \\
\text { Estadístic } \\
0\end{array}$} & \multicolumn{2}{|c|}{ Media } & \multirow{2}{*}{$\begin{array}{l}\text { Desv. típ. } \\
\text { Estadístic } \\
0\end{array}$} \\
\hline & & & & Estadístico & $\begin{array}{l}\text { Error } \\
\text { típico }\end{array}$ & \\
\hline Edad & 105 & 20,00 & 65,00 & 39,0667 & 1,11272 & 11,40198 \\
\hline $\begin{array}{l}\text { Tiempo } \\
\text { trabajado }\end{array}$ & 105 & ,20 & 38,00 & 12,2499 & ,93620 & 9,59318 \\
\hline
\end{tabular}

Las puntuaciones mínimas y máximas, medias y desviación típica en las diferentes dimensiones del engagement (vigor, absorción y dedicación) se detallan en la tabla 2:

Tabla 2. Estadísticos descriptivos de las dimensiones de vigor, absorción y dedicación del engagement.

\begin{tabular}{lccccc} 
& N & Mínimo & Máximo & Media & Desv. típ. \\
\hline VIGOR & 105 & 2,00 & 36,00 & 24,9333 & 5,73831 \\
ABSORCION & 105 & 4,00 & 36,00 & 22,4762 & 5,85579 \\
DEDICACION & 105 & 5,00 & 30,00 & 19,5238 & 6,22201 \\
\hline
\end{tabular}


Para el caso de la autoeficacia generalizada, los estadísticos descriptivos obtenidos son los siguientes:

Tabla 3. Estadísticos descriptivos de Autoeficacia generalizada.

\begin{tabular}{lccccc} 
& N & Mínimo & Máximo & Media & Desv. típ. \\
\hline GSE & 105 & 20,00 & 40,00 & 32,9238 & 3,83229
\end{tabular}

Para analizar la relación entre las diferentes dimensiones del engagement y con respecto a la variable de autoeficacia generalizada, se realizó un análisis de correlación de Pearson bivariada. En la Tabla 4 se pueden observar los resultados del análisis. Tal y como cabría esperar, las diferentes dimensiones del engagement correlacionan positivamente entre sí de forma bilateral; así VIGOR-ABSORCION tiene un coeficiente de correlación de $.768(p<.001)$, ABSORCION-DEDICACION, $r=.716$ $(p<.001)$ y DEDICACION-VIGOR, $r=.724(p<.001)$.

Por otro lado también existe una correlación positiva, aunque menos sólida que intradimensionalmente, entre la variable de autoeficacia generalizada y las distintas dimensiones del engagement, de forma bilateral, AUTOEFICACIA-VIGOR $r=.476$ $(p<.001), \quad$ AUTOEFICACIA-ABSORCION, $\quad r=.471 \quad(p<.001)$ y AUTOEFICACIADEDICACION, $r=.418,(p<.001)$.

Tabla 4. Coeficiente de correlación Pearson

\begin{tabular}{lrrrr} 
& GSE & VIGOR & ABSORCION & DEDICACION \\
\hline GSE & -- & & & \\
VIGOR &, $476^{* *}$ & -- & & \\
ABSORCION &, $471^{* *}$ &, $768^{* *}$ & -- & - \\
DEDICACION &, $418^{* *}$ &, $724^{* *}$ &, $716^{* *}$ & - \\
\hline
\end{tabular}

Nota: ${ }^{* *} p<.001$

\section{GSE= AUTOEFICACIA GENERALIZADA}

Los resultados de los componentes del engagement según el sexo del participante, el ámbito de la empresa y el nivel de estudios son los siguientes:

Tabla 5. Medias y desviaciones típicas de las dimensiones del engagement según el sexo de los participantes.

\begin{tabular}{|c|c|c|c|c|c|c|c|c|c|c|c|}
\hline \multicolumn{12}{|c|}{ SEGÚN EL SEXO } \\
\hline \multicolumn{4}{|c|}{ VIGOR } & \multicolumn{4}{|c|}{ ABSORCIÓN } & \multicolumn{4}{|c|}{ DEDICACIÓN } \\
\hline \multicolumn{2}{|c|}{$\mathrm{H}$} & \multicolumn{2}{|c|}{$M$} & \multicolumn{2}{|c|}{$\mathrm{H}$} & \multicolumn{2}{|c|}{ M } & \multicolumn{2}{|c|}{$\mathrm{H}$} & \multicolumn{2}{|c|}{ M } \\
\hline Media & DT & Media & DT & Media & DT & Media & DT & Media & DT & Media & $\mathrm{DT}$ \\
\hline 26 & 5.98 & 24.22 & 5.49 & 23.35 & 6.15 & 21.88 & 5.62 & 21,09 & 6,18 & 18,47 & 6,07 \\
\hline
\end{tabular}




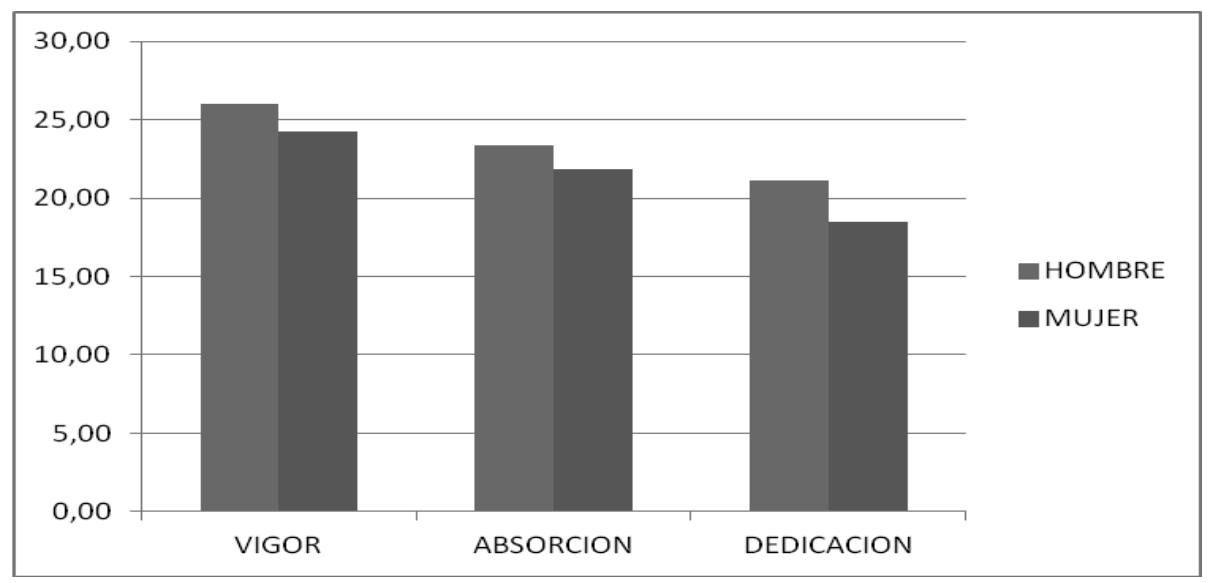

Gráfica 3. Comparativa de las medias de las dimensiones del engagement según el sexo.

Realizada la prueba $\mathrm{t}$ de Student para comparar las medias de las dimensiones del engagement teniendo en cuenta el sexo hemos obtenido los siguientes resultados. Para la dimensión VIGOR, se obtuvo un $\mathrm{t}(103)=1.566, \mathrm{p}=.120$, al ser éste mayor que 0.05 , no hay diferencia estadística entre las medias de los grupos de hombres y mujeres con respecto a esta dimensión. Para la dimensión ABSORCION, se obtuvo un $t(103)=1.262, p=.210$, al ser éste mayor que 0.05 , no hay diferencia estadística entre las medias de los grupos de hombres y mujeres con respecto a ABSORCION. Por último, para la dimensión DEDICACIÓN hemos obtenido un $t(103)=2.15, p=.034$, a diferencia de los casos anteriores, sí existe diferencia estadística entre las medias, con lo que éstas no serían equiparables.

Tabla 6. Medias y desviaciones típicas de las dimensiones del engagement según el ámbito laboral (público o privado) de los participantes.

\begin{tabular}{|c|c|c|c|c|c|c|c|c|c|c|c|}
\hline \multicolumn{12}{|c|}{ SEGÚN EL ÁMBITO } \\
\hline \multicolumn{4}{|c|}{ VIGOR } & \multicolumn{4}{|c|}{ ABSORCIÓN } & \multicolumn{4}{|c|}{ DEDICACIÓN } \\
\hline \multicolumn{2}{|c|}{ PÚBLICO } & \multicolumn{2}{|c|}{ PRIVADO } & \multicolumn{2}{|c|}{ PÚBLICO } & \multicolumn{2}{|c|}{ PRIVADO } & \multicolumn{2}{|c|}{ PÚBLICO } & \multicolumn{2}{|c|}{ PRIVADO } \\
\hline Media & DT & Media & DT & Media & DT & Media & DT & Media & DT & Media & DT \\
\hline 23,70 & 6,34 & 26,05 & 4,91 & 22,18 & 5,98 & 22,74 & 5,78 & 18,46 & 5,98 & 20,49 & 6,32 \\
\hline
\end{tabular}




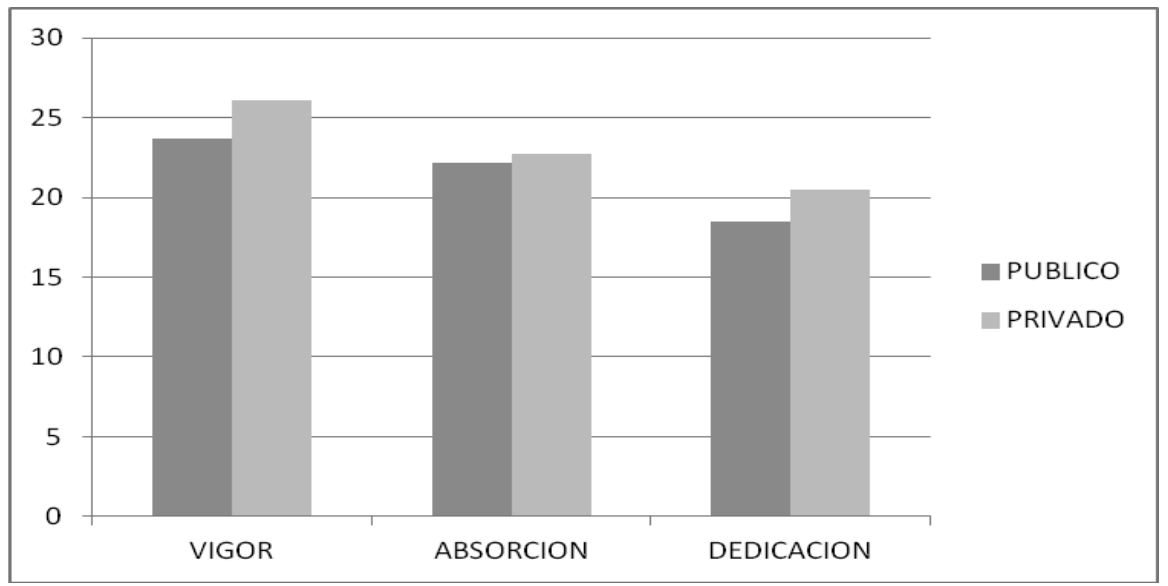

Gráfica 4. Comparativa de las medias de las dimensiones del engagement según el ámbito.

Realizada la prueba $t$ de Student para comparar las medias de las dimensiones del engagement teniendo en cuenta el ámbito laboral de los participantes (público o privado) hemos obtenido los siguientes resultados. Para la dimensión VIGOR, se obtuvo un $t(103)=-2.136, p=.035$, al ser éste menor que 0.05 , existe diferencia estadística entre las medias de los grupos público y privado a favor de este último. Para la dimensión ABSORCION, se obtuvo un $t(103)=-.492, p=.624$, al ser éste mayor que 0.05 , no hay diferencia estadística entre las medias de los grupos público y privado con respecto a $A B S O R C I O N$. Por último, para la dimensión DEDICACIÓN hemos obtenido un $t(103)=-1.685, p=.095$, en este caso no existe diferencia significativa de medias entre los grupos público y privado.

Tabla 7. Medias y desviaciones típicas de las dimensiones del engagement según el nivel de estudios de los participantes.

\begin{tabular}{|c|c|c|c|c|c|c|c|c|c|c|c|c|c|c|c|c|c|}
\hline \multicolumn{18}{|c|}{ SEGÚN NIVEL DE ESTUDIOS } \\
\hline \multicolumn{6}{|c|}{ VIGOR } & \multicolumn{6}{|c|}{ ABSORCION } & \multicolumn{6}{|c|}{ DEDICACION } \\
\hline \multicolumn{2}{|c|}{ BAS } & \multicolumn{2}{|c|}{ MED } & \multicolumn{2}{|c|}{ UNIV } & \multicolumn{2}{|c|}{ BAS } & \multicolumn{2}{|c|}{ MED } & \multicolumn{2}{|c|}{ UNIV } & \multicolumn{2}{|c|}{ BAS } & \multicolumn{2}{|c|}{ MED } & \multicolumn{2}{|c|}{ UNIV } \\
\hline M & DT & M & DT & $M$ & DT & M & DT & M & DT & M & DT & $\mathrm{M}$ & DT & $M$ & DT & M & DT \\
\hline $\begin{array}{c}25, \\
8\end{array}$ & $\begin{array}{c}3.3 \\
1\end{array}$ & $\begin{array}{c}24 \\
7\end{array}$ & $\begin{array}{c}6,4 \\
8\end{array}$ & $\begin{array}{c}24 \\
9\end{array}$ & $\begin{array}{c}5,5 \\
4\end{array}$ & $\begin{array}{c}22 \\
1\end{array}$ & $\begin{array}{c}5,2 \\
7\end{array}$ & $\begin{array}{c}21 \\
7\end{array}$ & $\begin{array}{c}6,3 \\
0\end{array}$ & $\begin{array}{c}22 \\
9\end{array}$ & $\begin{array}{c}5,6 \\
9\end{array}$ & $\begin{array}{c}19 \\
5\end{array}$ & $\begin{array}{c}5,5 \\
7\end{array}$ & $\begin{array}{c}19 \\
9\end{array}$ & $\begin{array}{c}5,9 \\
3\end{array}$ & $\begin{array}{c}19 \\
3\end{array}$ & 6,5 \\
\hline
\end{tabular}




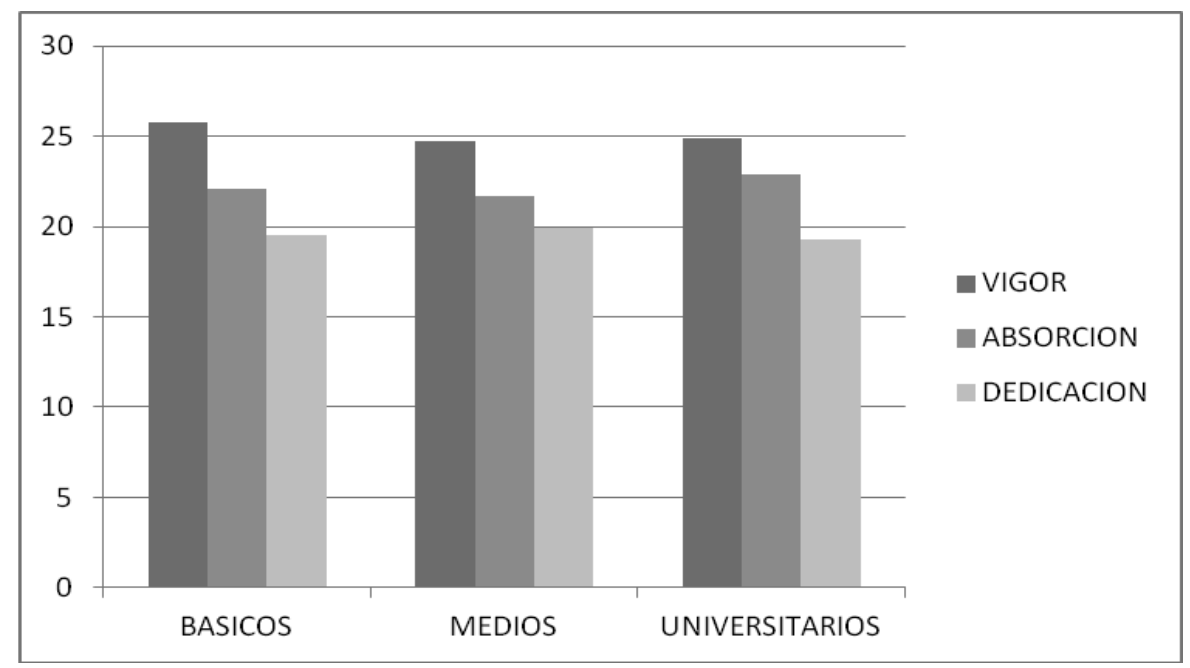

Gráfica 5. Comparativa de las medias de las dimensiones del engagement según el nivel de estudios.

Agrupando el nivel de estudios en universitario o no universitario, obtenemos los siguientes resultados para las dimensiones del engagement:

Tabla 8. Medias y desviaciones típicas de las dimensiones del engagement según si se tienen o no estudios universitarios.

\begin{tabular}{lllcc} 
& \multicolumn{1}{c}{ UNINO } & N & Media & Desviación típ. \\
\hline \multirow{2}{*}{ VIGOR } & NOUNIVERSITARIO & 41 & 24,9268 & 6,10488 \\
& UNIVERSITARIO & 64 & 24,9375 & 5,54026 \\
\hline \multirow{2}{*}{ ABSORCION } & NOUNIVERSITARIO & 41 & 21,7805 & 6,10538 \\
& UNIVERSITARIO & 64 & 22,9219 & 5,69406 \\
\hline \multirow{2}{*}{ DEDICACION } & NOUNIVERSITARIO & 41 & 19,8537 & 5,81619 \\
& UNIVERSITARIO & 64 & 19,3125 & 6,50488 \\
\hline
\end{tabular}

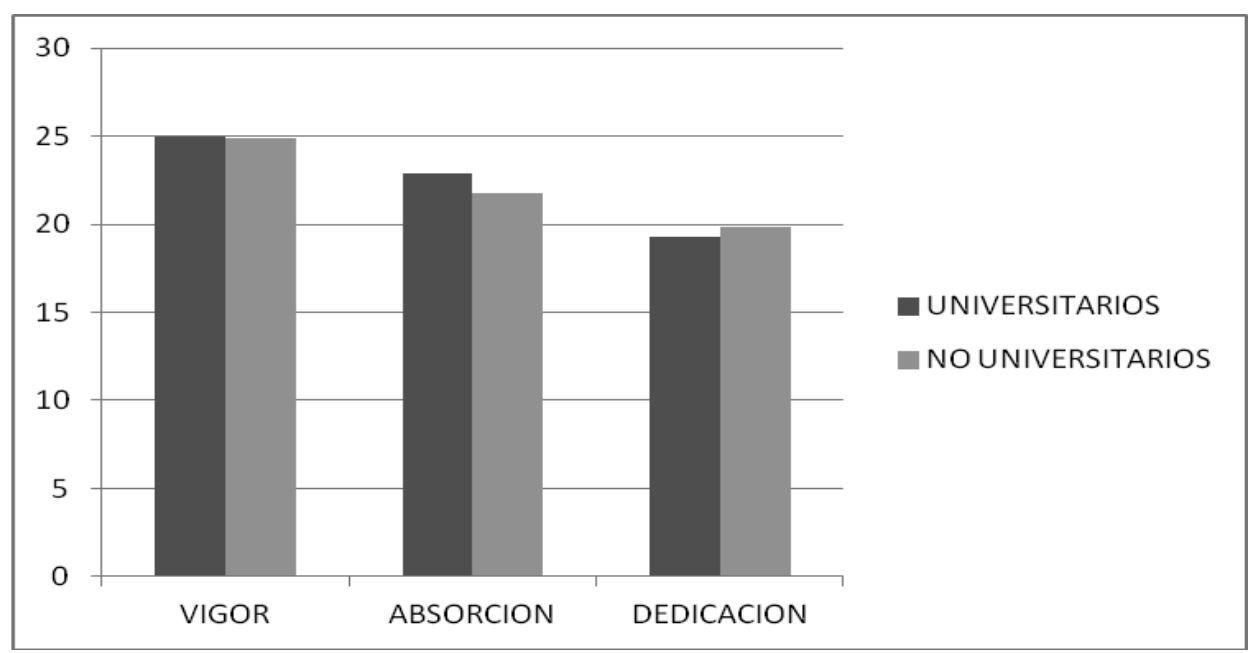

Gráfica 6. Comparativas de las medias de cada una de las dimensiones del engagement en población universitaria y no universitaria. 
Se realizó la prueba t de Student para comparar las medias de las dimensiones del engagement teniendo en cuenta si se tenían o no estudios universitarios obteniendo los siguientes resultados: para la dimensión VIGOR, se obtuvo un t $(103)=-.009, p=$ .993, lo que indica que no existen diferencias significativas entre las medias de los grupos con estudios universitarios y sin estudios universitarios. Para la dimensión ABSORCION, se obtuvo un $\mathrm{t}(103)=-.974, \mathrm{p}=.332$, de nuevo no hay diferencia estadística entre las medias de los grupos. Por último, para la dimensión DEDICACIÓN hemos obtenido un $\mathrm{t}(103)=.433, \mathrm{p}=.666$, tampoco existe diferencia significativa de medias entre los grupos con estudios universitarios y sin ellos.

\section{DISCUSIÓN}

En el presente estudio intentamos determinar si existe relación entre la Autoeficacia percibida y el compromiso organizacional (engagement) de un individuo con su contexto laboral, además de la dirección de esta relación (directa o inversa). A su vez, hemos intentado determinar si este nivel de Engagement se ve condicionado por variables como sexo, ámbito laboral y nivel de estudios.

Tras analizar los resultados comprobamos que existe una correlación positiva entre "Autoeficacia" y "Engagement", como planteábamos en nuestra primera hipótesis. Existe correlación positiva entre las dimensiones de "Engagement" (VIGOR, ABSORCIÓN y DEDICACIÓN) así como una correlación también positiva entre éstas y "Autoeficacia".

Tras realizar la comparación de datos entre hombres y mujeres observamos que las medias de las puntuaciones obtenidas en las dimensiones de VIGOR y ABSORCIÓN y DEDICACIÓN del engagement para el grupo de hombres fueron mayores que las de las mujeres. Sin embargo, la comparación de medias mediante la t-student arrojó los resultados de que en las dimensiones de VIGOR y ABSORCION, las diferencias no eran significativas, con lo que no existiría diferencia y se cumple nuestra hipótesis $\mathrm{H} 2$ para esas dimensiones. Sin embargo no ocurre así para la dimensión de DEDICACIÓN, en la que la diferencia de medias es significativa a favor del grupo de hombres.

Una posible explicación a esta diferencia se podría atribuir a diferencias de roles entre sexos aún presentes en nuestra sociedad. Por lo general, el hombre no tiene tantas responsabilidades fuera del ámbito laboral como la mujer, quien aparte de su puesto de trabajo, debe atender a labores domésticas o de educación. Esta menor carga de responsabilidad permitirían al hombre mayor focalización e inversión de esfuerzo en el puesto de trabajo.

Otra de las posibles variables que creímos que pudiera causar diferencias en cuanto a Engagement es el ámbito laboral, distinguiendo entre puestos públicos y privados. Los resultados obtenidos muestran que las medias de todas las dimensiones del engagemetn son más altas para el ámbito privado que para el público, sin embargo, sólo son significativas en la dimensión de VIGOR a favor del ámbito privado. Esto implica que nuestra hipótesis H3 no se cumpliría o sólo se cumpliría en la dimensión de VIGOR.

Mayor vigor en el sector privado lo asociamos a mayor responsabilidad por parte del trabajador, mayor exigencia y rendimiento en el puesto de trabajo.

Nuestra hipótesis respecto a la variable nivel de estudios, $\mathrm{H} 4$, apostaba por valores de 
Engagement más elevados para personas con niveles de estudios superiores, por encima de aquellos que no hubieran obtenido formación universitaria. Sin embargo, los resultados muestran que nuestra hipótesis no se cumple, en ninguna de las dimensiones del Engagement, al no existir diferencias significativas respecto a las medias de "universitarios" frente a "no universitarios".

Una posible explicación a estos resultados puede deberse a que muchos "universitarios" de nuestro estudio realizan trabajos que no se corresponden al nivel de las competencias adquiridas. Consideramos que si los empleos de los sujetos universitarios estuvieran relacionados con sus competencias académicas, la diferencia de medias hubiera sido significativamente mayor a favor de éstos.

Aunque los resultados obtenidos y su posterior interpretación confirman nuestra primera hipótesis $(\mathrm{H} 1)$ y parcialmente alguna de las demás, este estudio no está exento de limitaciones. Tal y como dicen las teorías organizacionales y diversos estudios, en nuestra muestra se puede aceptar que la "Autoeficacia" funciona como variable mediadora de "Engagement" ya que los datos muestran una correlación positiva entre ambos constructos. Sin embargo, la influencia de variables como sexo, nivel de estudios y ámbito laborales casi inexistente en la muestra de nuestro estudio.

Una de las limitaciones que nos hemos encontrado en la elaboración de este estudio es conseguir una muestra adecuada para el mismo. Uno de los requisitos de nuestra muestra debía consistir en personas que se encontraran trabajando actualmente y que estuvieran dispuestas a participar voluntariamente. En los tiempos de crisis que corren, no es tan fácil encontrar a gente con empleo, y aun encontrando a personas con estas características, algunas de ellas eran reticentes a la hora de participar, por si los datos se les suministraban a sus superiores.

Con respecto a la variable "ámbito laboral", se han seleccionado puestos de trabajo muy distintos entre sí. Si los empleos hubieran sido más homogéneos dentro de cada sector, quizá las diferencias en cuanto a la variable "ámbito laboral" habrían sido significativas en todas las dimensiones.

En cuanto a la variable "nivel de estudios" hay que tener en cuenta que los datos no sugieren ninguna diferencia entre "universitarios" y "no universitarios" respecto a puntuaciones en Engagement. Con un tamaño mayor de muestra, quizá estos resultados hubieran sido distintos a los obtenidos.

Por último y refiriéndonos a la variable "sexo", recordar que la muestra fue aleatoria y es posible que los empleos desempeñados por hombres tuvieran más capacidad de generar Engagement que aquellos realizados por mujeres, lo que explicaría mayores puntuaciones en la dimensión "dedicación" para el sector masculino.

Pensamos que en el ámbito laboral es muy importante conseguir que los trabajadores se encuentren comprometidos con la organización, es decir, conseguir un alto nivel de Engagement, ya que esto asegurará trabajadores más realizados con sus puestos de trabajo y al mismo tiempo más productivos y eficaces. Por ello es importante estudiar todas aquellas variables que puedan ayudar a favorecer esta situación y se abre un nuevo campo de investigación.

En este caso, se han estudiado variables como Autoeficacia, sexo, ámbito o nivel de estudios. En un futuro, se podrían estudiar otras variables susceptibles de mediar en el Engagement tales y como autoestima, clima laboral, condiciones laborales... 


\section{REFERENCIAS BIBLIOGRÁFICAS}

Bakker, A. y Oerlemans, W. (2011). Subjective well-being in organizations. En K. Cameron Y G. Spreitzer (Eds), Handbook of positive organizational scholarship. New York: Oxford University Press.

Bandura, A. (2002). Social cognitive theory in cultural context. Journal of applied Psychology: An International Review, 51, 269-290.

Bandura, A. (2004). Health promotion by social cognitive means. Health, Education \& Behavior, 31 (2), 143-164

Cherniss , C. (1980). Role of professional self-efficacy in the etiology and amelioration of burnout. En W.B. Schaufeli, C. Maslach y T. Marek (Eds.), Professional burnout: Recent developments in theory and research (pp, 135-150). Washington, DC: Taylor \& Francis.

Cherniss , C. (1993). Role of professional self-efficacy in the etiology and amelioration of burnout. En W.B. Schaufeli, C. Maslach y T. Marek (Eds.), Professional burnout: Recent developments in theory and research. Series in applied psychology: Social issues and questions (pp, 135-149). Washington, DC: Taylor \& Francis.

Luthans, F., Youssef, C. y Avolio, B. (2007). Psychological capital: Developing the human competitive edge. Oxford, United Kingdom: Oxford University Press.

Manzano, G. (2002). Burnout y engagement en un colectivo profesional. Boletín de Psicología, 74, 79-102.

Macey, W. y Schneider, B. (2008). The meaning of employee engagement. Industrial and Organizational Psychology: Perspectives on Science and Practice, 1, 3-30.

Martínez, I. y Salanova, M. (2006). Autoeficacia en el trabajo: el poder de creer que tú puedes. EstudiosFinancieros, 279 (45), 175-202.

Salanova, M., Martínez, I. M., Llorens, S. (2006). Psicología Organizacional Positiva. In F.J. Palací (Coord.).Psicología de la Organización (349-376). Madrid: Prentice Hall.

Salanova, M. y Schaufeli, M. (2009). El engagement en el trabajo. Cuando el trabajo se convierte en pasión. Anales de psicología, 25 (2).

Salanova, M., Schaufeli, W.B., Llorens, S., Peiro, J.M., Grau, R. (2000). Desde el "burnout" al "engagement": ¿una nueva perspectiva?. Revista de Psicología del Trabajo y las Organizaciones. 16 (2) 117-134.

Schaufeli W. y Salanova M. (2011). Commentary work engagement: On how to better catch a slippery concept. European Journal of Work and Organizational Psychology, 20 (1), 39-46.

Schaufeli W., Salanova M., González-Romá, M., y Bakker A. (2002). The measurement of burnout and engagement: A confirmatory factor analytic approach. Journal of Happiness Studies, 3, 71-92.

Seligman, M., Steen, T., Park, N., y Peterson, C. (2005). Positive psychology practice: Empirical validation of interventions. American Psychologist, 60, 410-421.

Ventura, M., Llorens, S., Salanova, M .El rol de la autoeficacia en el estudio del engagement. Jornades de Foment de la Investigació. Universitat Jaume I. Extraída el 5 
de abril de 2013 de http://www.uji.es/bin/publ/edicions/jfi12/4.pdf. 